\title{
ELECTRONOGRAPHIC PHOTOMETRY OF STARS IN THE GLOBULAR CLUSTERS OF THE MAGELLANIC CLOUDS
}

\author{
M. F. WALKER
}

Lick Observatory, Calif., U.S.A.

\begin{abstract}
Due to the linearity, high quantum efficiency, and high storage capacity of electronographic image intensifiers, their application to astronomical photometry constitutes an advance equal in importance to those that resulted from the introduction of the photographic plate and the photomultiplier. With existing electronographic image tubes and telescopes of 60 -in. aperture stellar photometry is possible to about magnitude 23. Consequently a major advance in the study of the stellar contents of galaxies in the Local Group may now be made.

During 1968-69, the spectracon image intensifier was used on the 60-in. reflector of the Cerro Tololo Observatory for B, $\mathrm{V}$ observations of 14 globular clusters in the Magellanic Clouds. Details of the observing program and discussions of the observations so far reduced will appear shortly in papers in the Astrophysical Journal and ir. Sky and Telescope.
\end{abstract}

